\title{
The Computation of Position Sense from Spindles in Mono- and Multiarticular Muscles
}

\author{
S. H. Scott ${ }^{\mathrm{a}}$ and G. E. Loeb \\ MRC Group in Sensory-Motor Physiology and Bio-Medical Engineering Unit, Queen's University, Kingston, Ontario K7L \\ 3N6 Canada
}

It is known that muscle spindles provide the majority of information about limb position, but little is known about how position sense is computed from their signals. We have developed a family of musculoskeletal models in order to determine some of the fundamental properties associated with transforming noisy spindle information into putative internal coordinate frames for position sense. A two-joint model was developed containing one biarticular and two monoarticular muscles with a total of 1000 sensors distributed among them. The sensors were assumed to function like spindle secondary afferents under fusimotor control designed to optimize their ability to encode static position in the presence of constant output noise. The optimal distribution of sensors was found to depend strongly on the coordinate frame in which position was measured (intersegmental angle, segment orientation, or end-point of the limb) and on the topology of the biarticular muscle with respect to the plane of motion. A similar analysis was performed for an anthropometric model of the human arm, using previously published counts of muscle spindles. In general, the actual distribution of spindles about the elbow and shoulder does not seem to favor any single coordinate frame for position sense. We also looked at the potential accuracy in detecting changes in joint angles based on the distribution of muscle spindles throughout the human body. The distribution of spindles about individual joints accounts well for psychophysical data showing a proximodistal descending gradient of angular resolution that partially reflects the relative importance of more proximal joints for determining the location of the end-point.

[Key words: posture, position sense, kinesthesia, modeling, muscle spindle, multiarticular]

To plan and execute voluntary limb movements to attain specific postures or targets, the CNS must have a way to represent the current position of the limb in relation to the desired posture or location of the target. The goal may be specified by current sensory information from any of several different modalities or by memory from an internal representation. Each of these sources of information has a different, natural coordinate frame based

\footnotetext{
Reccived Aug. 31, 1993; reviscd May 23, 1994; acceptcd June 2, 1994.

This work was supported by the Canadian MRC Group in Sensory-Motor Physiology. We thank Drs. V. C. Abrahams, D. P. Munoz, and F. J. R. Richmond for valuable comments on the manuscript.

Correspondence should be addressed to Dr. G. E. Loeb, Abramsky Hall, Queen's University, Kingston, Ontario K7L 3N6 Canada.

aPresent address: Department of Physiology, University of Montreal, Montreal, Quebec H3C 3J7 Canada.

Copyright (c) 1994 Society for Neuroscience $0270-6474 / 94 / 147529-12 \$ 05.00 / 0$
}

on its intrinsic properties (e.g., retinotopic map, interaural amplitude and timing, skin surface map, etc.). Furthermore, none of these frames is identical with or even easily transformed into the output coordinate frame represented by the complex, nonorthogonal set of skeletal muscles and their mechanical actions. Thus, it seems likely that the CNS explicitly computes at least one internal representation of body posture and target position in a coordinate frame that is intermediate between the various intrinsic input and output coordinate frames.

Several possible orthogonal coordinate frames have been proposed for this intermediate representation (reviewed by Soechting and Flanders, 1992). Intersegmental joint angle (herein designated $\phi$ ) is most commonly used for the control of multiarticulated robots and has some biological appeal because of the distribution of proprioceptors in muscles, ligaments, and joint capsules that span individual joints. Absolute segmental orientation (herein designated $\theta$ ) with respect to an external inertial frame or the axis of the trunk has been suggested based on psychophysical performance of human subjects asked to match particular postures (Soechting and Ross, 1984; Mouchnino et al., 1993). Several interrelated frames based on external, cartesian coordinates are possible, including absolute end-point location and location relative to current posture, each of which could be based upon any of several plausible locations for the origin (e.g., head centered, shoulder centered, trunk centered). An external coordinate frame is often implicit in the design of reaching tasks and in the interpretation of neurophysiological data obtained during these tasks, such as tuning curves of unit recordings from cerebral cortical cells (Georgopoulos et al., 1986; Caminiti et al., 1991). In addition to these various "convenient" choices, an infinity of other coordinate frames is mathematically possible, most having no one-to-one correspondence with intuitively simple or experimentally measurable variables. It also seems likely that any coordinate frame will have both static and dynamic aspects, perhaps represented separately, corresponding to the senses of posture (position sense) and kinesthesia (velocity sense), respectively.

Rather than attempting to guess which coordinate frame might be employed by the CNS, we have asked whether any clues can be found in the structure of the musculoskeletal system itself. The presumption is that errors in posture sense and kinesthesia would be a limiting factor in motor performance, which in turn would result in strong evolutionary pressure to minimize such errors. If limited sensory resolution is a contributing factor to such errors, evolutionary pressure should result in changes in the numbers and distributions of the various types of sensors available for computing posture and kinesthesia. In fact (as discussed below), individual proprioceptors are quite noisy, their 
numbers are finite, their forms are highly evolved, and they are distributed in an anatomically specific manner that is consistent with such performance-driven evolution toward an optimized distribution. We show here that the optimal distribution of noisy sensors in limbs with both uni- and multiarticular muscles depends strongly on the coordinate frame in which computations are performed and accuracy is assessed.

The nature of such models can be simplified greatly by the recent recognition that the muscle spindles are generally the limiting factor in the precision of proprioception (except perhaps in the digits), with joint receptors and skin stretch receptors playing only a secondary role when spindle information is absent or severely degraded (for review, see Gandevia et al., 1992). Fortunately, there exists a remarkably complete accounting of the numbers of spindles in virtually all of the muscles of the human (Voss, 1971). Furthermore, there is considerable circumstantial evidence to suggest that the design and distribution of spindles among and within individual muscles are not random, but are highly specific, constant among individuals, and thus likely to be genetically determined (Voss, 1971; Matthews, 1972; Banks and Stacey, 1988). Because many of the receptors reside in muscles that cross more than one joint, we have developed a musculoskeletal model that computes limb position in any coordinate frame from all available sensors. From the distribution of sensors and the assumption that the CNS makes optimal use of all available information, we have computed the errors that would be expected in various reference frames and postures.

We further postulate that the musculoskeletal system of humans is indeed highly evolved to perform precise motor tasks and that such evolution has resulted in nearly optimal distributions of muscle spindles in the various muscles. The apparent accuracy of the actual distribution of spindles acting about each joint throughout the body was assessed and compared to psychophysical data regarding angular resolution. We then asked whether the actual distribution is more consistent with computation in any one of the various coordinate frames that have been suggested for the common, internal representation in the CNS. A similar sort of "inverse analysis" has been used with some success in paleontology to make inferences about habitat and behavior from their apparent evolutionary effects on the mechanical properties of fossil skeletons (Thompson, 1917). Neuroscientists usually take the peripheral sensorimotor apparatus as a given, concentrating instead on the evolution of the cognitive apparatus. For proprioception, which is phylogenetically old and central to the daily survival of animals, we suggest that significant coevolution must have occurred and that the present form of the peripheral apparatus may provide valuable and readily accessible clues to the nature of the central processor that receives its signals.

\section{Materials and Methods}

\section{Spindle model}

Mammalian muscle spindles are complex, pleiomorphic structures whose receptor properties are modulated strongly and continuously by several types of fusimotor control (reviewed by Loeb, 1984). Various mathematical models have been developed to describe the gain control of this transduction process (Hasan, 1983; Schaafsma et al., 1991). To develop quantitative models of the information-carrying capacity of large ensembles of these sensors, we have made a number of simplifying assumptions about their properties and function.

Static versus dynamic models. We have confined our models and tasks to static limb position rather than dynamic or kinesthetic sensation.
Spindles generally are innervated by two types of afferent neurons: type Ia fibers, forming primary endings near the midpoint of all of the intrafusal muscle fibers, and type II fibers, forming flanking secondary endings on only a subset of intrafusal muscle fibers (reviewed by Hulliger, 1984). The secondary afferents are sensitive mostly to static length, whereas primary afferents are sensitive to a mix of length and velocity; the mix depends strongly on the nature of the fusimotor drive and the amount and history of stretch. In the simplest terms, our model assumes that spindle output is linearly related to the length of the sensory portion of the spindle and is consistent with the notion that static limb position is computed exclusively from signals generated by the static secondary endings. However, it is computationally possible and it seems likely that the CNS uses information from the primary afferents as well, possibly by integrating their velocity information and/or by deconvolving the length information from their composite signal (see Matthews, 1988). Assuming that such computations are applied similarly to all muscle spindles, this would not affect the general conclusions drawn from our models. It is also possible that the CNS explicitly computes a dynamic (kinesthetic) coordinate frame in which the axes are the temporal derivatives of the axes of the static (position) coordinate frame. Position sense and kinesthesia have been separated psychophysically (Clark et al., 1986), although the neuronal correlates of these two senses do not appear to be segregated, at least in primary somatosensory cortex (Prud'Homme and Kalaska, 1994). Given reasonable assumptions, the general conclusions of our static model would probably extend to the dynamic frame, but we have not explored this explicitly.

Fusimotor control. Our models assume that muscle spindles are general-purpose sense organs whose output signals and dynamic gain control (intrafusal motor innervation) can be adapted by the CNS to meet the needs of a wide range of cognitive and motor tasks. In particular, we employ a global strategy for determining the appropriate "fusimotor set" for the spindles of each muscle, in which the dynamic range of the receptor sensitivity is modulated to coincide with the anticipated dy. namic range of mechanical stimuli associated with the task. For the task analyzed here, which consists of identifying body position, this translates into the simple notion that no sensor will reach saturation (i.e., a firing rate that is too low or too high to provide useful incremental information) and that all sensors will be modulated identically. The historical basis and further implications of this notion are considered in the Discussion.

Noise. One obvious role for the evolution of independently controllable fusimotor neurons would be to optimize the resolution of the received information in the face of noise in the neural signal itself (Loeb, 1984). The effect of noise can be minimized by the above-noted tuning of dynamic range (although not without some added noise produced by the fusimotor system itself). The noise itself arises inevitably in the process of converting continuous generator potentials from several, separate transduction sites in each ending into a train of frequency modulated, all-or-none action potentials. It can be filtered centrally by one of two processes (Stein, 1967): temporal integration, which reduces the rate at which changes in mechanical input can be appreciated (bandwidth), or spatial integration, which reduces the ability to resolve differences in mechanical conditions among muscles and their compartments. These factors have been analyzed quantitatively for the signals from muscle spindle afferents (Loeb and Marks, 1985); even with careful control of fusimotor set, the numbers of spindles actually found in individual muscles seemed in general to provide surprisingly limited resolution of muscle length and velocity.

In this report, we have extended this analysis to predict the resolution of limb position that would be obtained from the complete ensemble of spindles in the human arm. The accuracy of human spindles can be deduced from microneurographic recordings from individual spindle afferents during controlled conditions. The standard deviation of the spindle error was assumed to equal 0.5 radian based on experimental work by Vallbo (1974). This trial-to-trial variability in the spindle output signal was applied to the spindles in all muscles in the model according to the assumption above regarding a universal strategy for fusimotor control.

Signal channels. We have assumed that each spindle represents one information channel and that all information channels are identical. Unfortunately, mammalian spindles in general and human spindles in particular are quite pleiomorphic, with a wide range of numbers and types of sensory endings in each spindle. Individual spindles usually have at least two secondary afferents but may have as many as six (Richmond et al., 1986). Almost all spindles have a single primary 

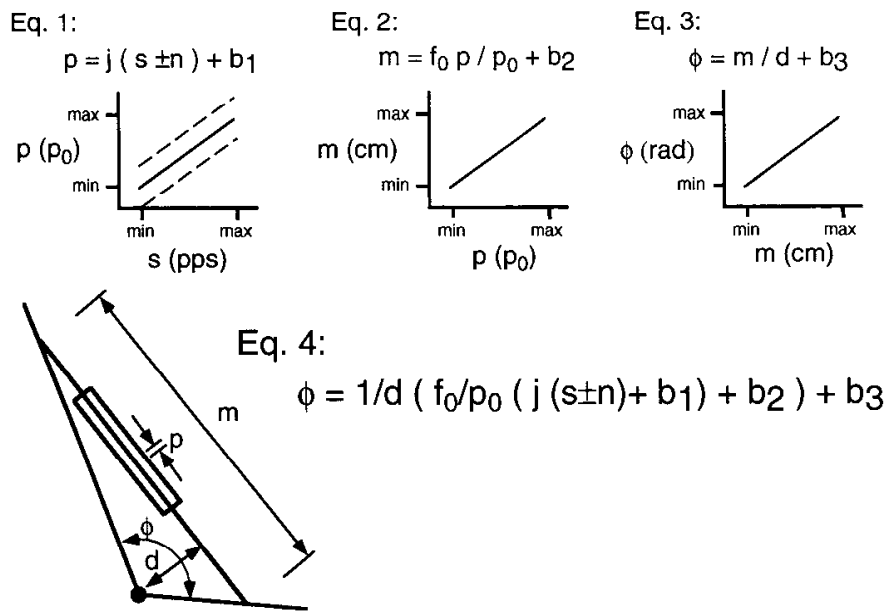

Figure 1. Linear model used to estimate the relationships between muscle spindle firing rate $(s)$ and length of the sensory region $(p)$, muscle fascicle length $(m)$ and joint angle $(\phi)$, whereby the nervous system can cornpute position sense from spindle activity.

ending, but there are variations in the numbers and types of intrafusal muscle fibers that result in changes in their receptor properties even in the absence of fusimotor input (Matthews, 1972; Richmond et al., 1986). From the few muscles in which there are some data about distribution of spindle forms, there seems to be no particular tendency toward an inverse relationship between spindle numbers or density and spindle complexity, suggesting that gross spindle counts provide a useful first approximation of the signal resolution obtainable from each muscle.

In summary, we model each individual spindle as a single channel of activity whose output $(s)$ is directly proportional to the length of its sensory part $(p)$ plus added noise $(n)$ :

$$
s=i p+b \pm n
$$

where $i$ is a proportionality constant and $b$ is a biasing constant (after Vallbo, 1974). We assume that the fusimotor system adjusts all spindles identically. We assume that the noise introduces an uncertainty in the CNS regarding the true length of the spindle that is constant over the entire range of length and firing rates. This constant uncertainty is intended to approximate the net effect of various stochastic processes in transduction, spike initiation, and postsynaptic integration, some of which produce noise that is proportional to firing rate and others of which produce noise that is inversely proportional to firing rate (Loeb and Marks, 1985). To describe the inverse process whereby the nervous system infers mechanical inputs from sensor outputs, it is useful to invert Equation 1 to

$$
p=j(s \pm n)+b_{1},
$$

where $j=1 / i$ and $b_{1}=-b / i$.

\section{Limb models}

It is necessary to consider how various aspects of musculoskeletal architecture (e.g., fascicle length, moment arm) affect the relationship between joint angle and the length of the sensory part of the spindle. For monoarticular muscles, this transformation can be broken down into two simple steps that scale the spindle output based on the musculoskeletal architecture. The spindle length corresponding to a certain whole-muscle length, $m$, will depend on the ratio between the fascicle length and spindle sensory length, based on

$$
m=f_{0} p / p_{0}+b_{2},
$$

where $f_{0}$ and $p_{0}$ correspond to the "resting" fascicle and spindle sensory lengths, respectively. These lengths are usually defined as the lengths where maximal force is generated by the muscle. The final transformation (assumed linear in this model) from muscle length to joint angle $(\phi)$ in radians is described by

$$
\phi=m / d+b_{3}
$$

MODEL 1

MODEL 2
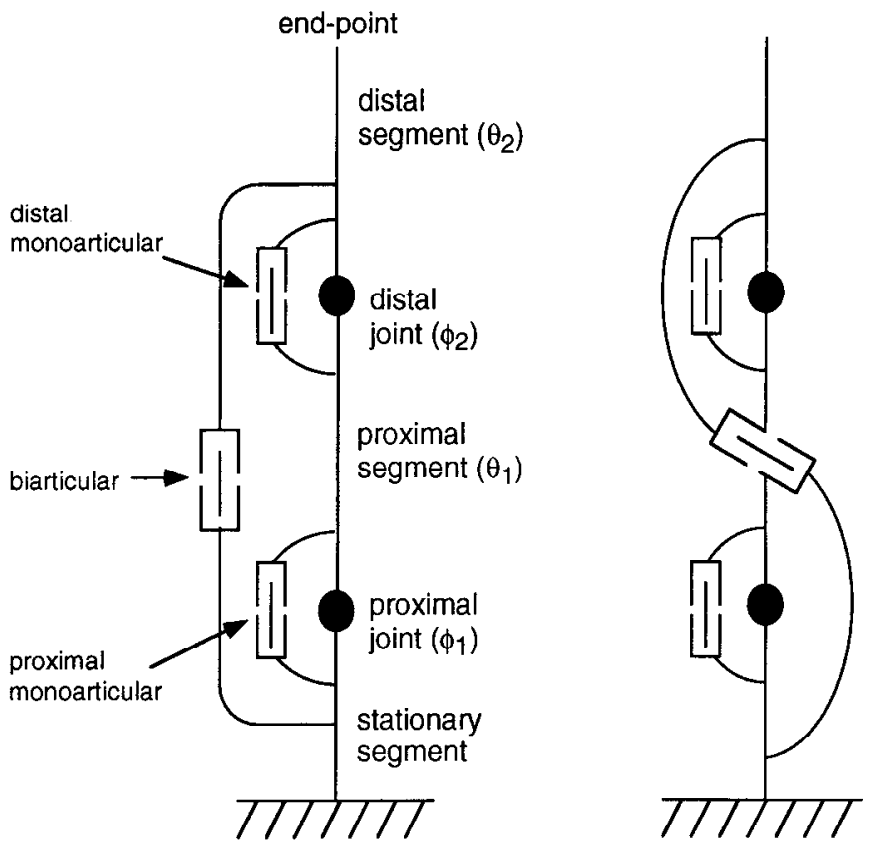

Figure 2. Two topologically distinct musculoskeletal models for a threemuscle, two-joint system.

where $d$ is the moment arm of the muscle, the shortest distance between the joint center and the line of action of the muscle. Figure 1 shows the relationships and combined function of Equation 4 from Equations 1-3:

$$
\phi=1 / d\left(f_{0} / p_{0}\left(j(s \pm n)+b_{1}\right)+b_{2}\right)+b_{3}
$$

We are interested in the precision rather than the absolute accuracy of static position sense; indeed, there is evidence that the sense of position derived from proprioception drifts rather badly if not recalibrated frequently by visual input (Wann and Ibrahim, 1992). Because precision requires only a relative and not an absolute relationship between spindle activity and joint angle, the transformations in Equations $1-3$ can be simplified to give

$$
\Delta s=\Delta \phi / k,
$$

where $k=\left(f_{0} j\right) /\left(p_{0} d\right)$ and $\Delta s$ and $\Delta \phi$ are the change in spindle activity and joint angle, respectively. Note that in $k$, two of the parameters, $j$ and $p_{0}$, describe properties intrinsic to the spindle, while $f_{0}$ and $d$ are properties of the musculoskeletal system.

For multiarticular muscles, spindle activity cannot be related directly to a single joint angle. In the case of biarticular muscles, the relationship between spindle activity and the orientation of the two spanned joints, 1 and 2 , is

$$
\Delta s=\left(\Delta \phi_{1} / k_{1}\right)+\left(\Delta \phi_{2} / k_{2}\right)
$$

Therefore, spindle activity in a biarticular muscle cannot directly describe any relative angle or position within the body. A change in spindle activity may result from movement of either of the spanned joints. There will be no change in activity if movement at one joint that shortened the muscle was counterbalanced by an opposing movement of the other spanned joint that lengthened the muscle.

We developed a simple musculoskeletal model to look at the role of spindles within these multiarticular muscles (Fig. 2). The model contains three segments: a stationary, a proximal, and a distal segment. These segments are connected by two joints, proximal $\left(\phi_{1}\right)$ and distal $\left(\phi_{2}\right)$. Each joint is spanned by a monoarticular muscle plus a biarticular muscle that spans both joints. The estimation of joint angle was described by

$$
\begin{array}{ll}
\Delta \phi_{1}=w_{1} k_{1} \Delta S_{1}+w_{2}\left(k_{2} \Delta S_{2}-\Delta \phi_{2} k_{2} / k_{4}\right), & w_{1}+w_{2}=1, \\
\Delta \phi_{2}=w_{3} k_{3} \Delta S_{3}+w_{4}\left(k_{4} \Delta S_{2}-\Delta \phi_{1} k_{4} / k_{2}\right), & w_{3}+w_{4}=1,
\end{array}
$$

where $S$ s are the averaged response from the ensemble of sensors in 
Table 1. Spindle counts in human muscles

\begin{tabular}{|c|c|c|c|c|c|}
\hline \multirow[b]{2}{*}{ Joint } & \multirow[b]{2}{*}{ DOF } & \multirow{2}{*}{$\begin{array}{l}\text { Distance to } \\
\text { end effector }(\mathrm{cm})\end{array}$} & \multicolumn{3}{|l|}{ Spindle Counts } \\
\hline & & & Monoarticular & Biarticular & Triarticular \\
\hline Upper cervical & 2 & 12 & 999 & 3302 & \\
\hline Lower cervical & 3 & 24 & $2311(?)$ & 94 & \\
\hline Sternoclavicular & 2 & 100 & 841 & $\begin{array}{r}94 \\
818\end{array}$ & \\
\hline Shoulder & 3 & 77 & 549 & $\begin{array}{l}818 \\
542\end{array}$ & \\
\hline Elbow & 2 & 45 & $785(812)$ & $\begin{array}{l}342 \\
304(324)\end{array}$ & \\
\hline Wrist & 2 & 19 & 333 & 314 & 356 \\
\hline Thumb & 3 & & 253 & 68 & \\
\hline Finger 1 & 2 & 7 & 101 & & \\
\hline Finger2 & 2 & & 91 & 545 & \\
\hline Finger 3 & 2 & & 63 & 53 & \\
\hline Finger 4 & 2 & & $42(163)$ & & \\
\hline Lumbar & 3 & 122 & $4524(?)$ & 656 & \\
\hline Hip & 3 & 108 & 2532 & $\begin{array}{l}000 \\
922\end{array}$ & \\
\hline Knee & 2 & 65 & 1262 & $\begin{array}{l}722 \\
195\end{array}$ & \\
\hline Ankle & 2 & 22 & 1118 & 244 & \\
\hline Great toe & 2 & & $220(266)$ & 244 & \\
\hline Toe 1 & 1 & 6 & 78 & & \\
\hline Toe2 2 & 1 & & 87 & $\begin{array}{l}310 \\
194\end{array}$ & \\
\hline Toe3 & 1 & & 84 & & \\
\hline Toe4 & 1 & & 123 & & \\
\hline
\end{tabular}

Data are a compilation of spindle counts in human muscles, according to which joints are crossed by each muscle and the distance from that joint to the end of the appendage, assuming a body-centered coordinate frame as shown in Figure 2. Fingers and toes refer to metacarpophalangeal and metatarsophalangeal joints only. Values in parentheses indicate alternative values in the literature or uncertainty due to the inclusion of muscles with multiple attachment points, only some of which span the joint in question.

each muscle and ws are weighting factors applied to the signals from these sensors. Subscripts 1 and 3 denote the proximal and distal monoarticular muscles, respectively. Subscripts 2 and 4 denote the biarticular muscle. These two equations can be separated to give

$$
\begin{aligned}
\Delta \phi_{1}= & \left(w_{1} k_{1} \Delta S_{1}+w_{2} k_{2} \Delta S_{2}-w_{2} w_{3} k_{3} \Delta S_{3} k_{2} / k_{4}\right. \\
& \left.-w_{2} w_{4} k_{2} \Delta S_{2}\right) /\left(1-w_{2} w_{4}\right), \\
\Delta \phi_{2}= & \left(w_{3} k_{3} \Delta S_{3}+w_{4} \mathrm{k}_{4} \Delta S_{2}-w_{4} w_{1} k_{1} \Delta S_{1} k_{4} / k_{2}\right. \\
& \left.-w_{4} w_{2} k_{4} \Delta S_{2}\right) /\left(1-w_{4} w_{2}\right) .
\end{aligned}
$$

It is interesting to note that the computation of either joint angle requires information explicitly from the nonspanning monoarticular muscle. Information on the nonspanning muscle is, of course, required in order that information from the biarticular can contribute to the estimate of joint angle, but what is the cost of this increased complexity in estimating joint angle or any other position parameter?

We looked at the information-based properties of sensory signals by looking at the susceptibility of the model to transmit noisy spindle information into different, putative reference frames that may describe limb posture. The noise transmitted to joint angle space could be determined directly from Equations 9 and 10 . The error transmitted to segment-angle reference frames, $\theta_{1}$ and $\theta_{2}$, was also tested based on

$$
\begin{gathered}
\Delta \theta_{1}=\Delta \phi_{1}, \\
\Delta \theta_{2}=\Delta \phi_{1}+\Delta \phi_{2} .
\end{gathered}
$$

The error transmitted to the end of the distal segment, end effector, was also calculated using

$$
\begin{aligned}
& x=L_{1} \cos \left(\phi_{1}{ }^{*}+\Delta \phi_{1}\right)+L_{2} \cos \left(\phi_{1}{ }^{*}+\phi_{1}+\phi_{2}{ }^{*}+\Delta \phi_{2}\right), \\
& y=L_{1} \sin \left(\phi_{1}{ }^{*}+\Delta \phi_{1}\right)+L_{2} \sin \left(\phi_{1}{ }^{*}+\Delta \phi_{1}+\phi_{2}{ }^{*}+\Delta \phi_{2}\right),
\end{aligned}
$$

where $L_{1}$ and $L_{2}$ are the lengths of the proximal and distal segments, respectively, and $\phi_{1}{ }^{*}$ and $\phi_{2}{ }^{*}$ are the initial joint angles for the proximal and distal joints, respectively. The total error at the end effector was the resultant vector of the cartesian coordinate errors $x$ and $y . L_{1}$ and $L_{2}$ were both set equal to $30 \mathrm{~cm}$ while the segment angles were varied to specify $10 \mathrm{~cm}$ increments along a vertical axis from full extension to $10 \mathrm{~cm}$ in front of the body (see Fig. 11).

Equations 9 and 10 describe the relationship of three muscles acting about two joints when the biarticular muscle spans the muscle on the same side of both joints (model 1 of Fig. 2, and topologically similar variants not shown). There is an alternate model where the biarticular spans opposite sides of each joint (model 2 of Fig. 2, and topologically similar variants not shown). The equations for this form of the model were also derived and the errors from noisy spindle data were transformed into the selected reference frames.

Each model had a total of 1000 spindles, giving rise to the triangular space, of all possible spindle distributions among three muscles, that is used for the contour plots (see Figs. 3-5, 10). Each spindle was assumed to have noise that was characterized by the standard, normal gaussian distribution. The transformation coefficients $\left(k_{1}, k_{2}, k_{3}\right.$, and $\left.k_{4}\right)$ were initially set to 1 so that noise transmission was similar for all muscles. The number of spindles in each muscle was systematically varied in increments of 50 . Note, however, that although a muscle may contain no spindles, a single muscle could not contain all 1000 spindles (or the model would have an indeterminate solution). The weighting for each muscle was set as a linear function of the total number of spindles that spanned the joint. For example, $w_{1}$ would equal 0.25 if the proximal monoarticular contained 100 spindles and the biarticular contained 300 spindles. The noise transmitted by the population of spindles in a muscle is equal to a standard, normal gaussian distribution with a variance equal to the variance of the noise for each individual spindle divided by the square root of the number of spindles within the muscle (Hogg and Tanis, 1977). Therefore, a random number was selected to define the error for the entire population of spindles within a muscle using an approximation to the normal distribution function. These errors were then transformed to each reference frame, using Equations 9-14. One thousand independent trials were performed for each distribution of spindles, and the standard deviation of the error transmitted to each reference frame was calculated. 


\section{JOINT ( $\Phi)$ AND SEGMENT ( $(\Theta)$ ERRORS FOR DIFFERENT SPINDLE DISTRIBUTIONS}
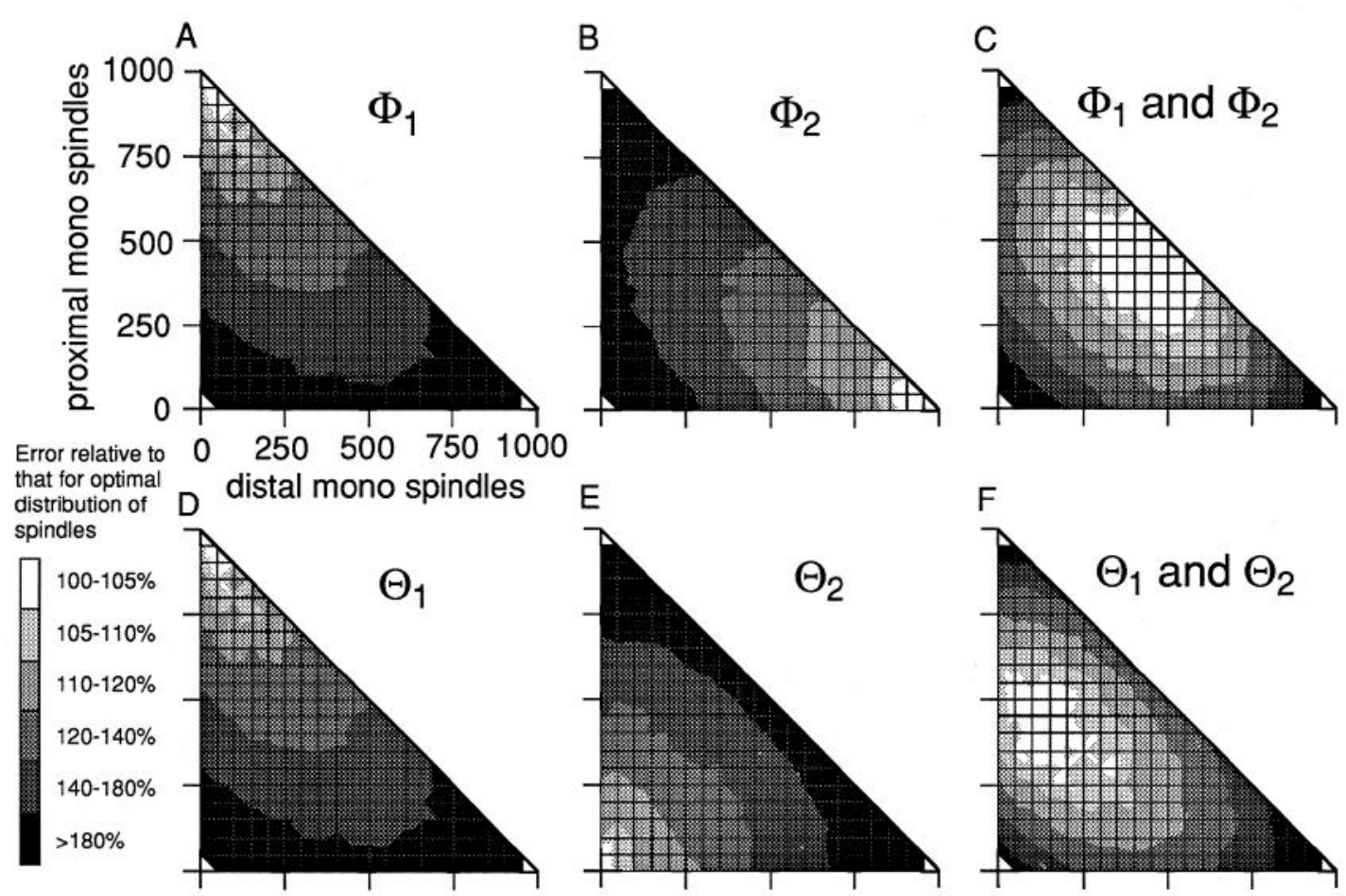

Figure 3. Contour plots depict relative position error produced by various distributions of spindles among the three muscles of the idealized twojoint system shown in Figure 2 (model 1). The total number of spindles is fixed (to 1000 here). The diagonal border represents distributions with no biarticular spindles; points closer to the origin represent increasing numbers of spindles in the biarticular muscle. The lighter regions of the contour plots represent distributions that produce lower errors when used to estimate the value of the particular angular coordinate that is labeled in each plot.

\section{Human spindle distributions}

An alternate way to learn about the utility of spindles for determining position sense is to look at the actual distribution of spindles in the body. A study by Voss (1971) determined the number of spindles and the weight of almost all skeletal muscles. Added to this database are spindle counts by Amonoo-Kuofi (1983) for some of the axial muscles not enumerated by Voss. We counted the number of spindles that spanned the major joints (or joint systems) in the human body. The number of spindles from each muscle was distributed equally among all spanning joints. If the muscle spanned only one joint, that joint received all of the muscle's spindles. If the muscle spanned three joints, each joint received one-third of that muscle's spindles. Table 1 shows the distribution of spindles in terms of the joints that their parent muscles cross.

The signal-to-noise ratio that can be extracted from a group of independent sources increases with the square root of their number (Hogg and Tanis, 1977). Therefore, the square root of the total number of spindles spanning a joint was used to estimate the potential accuracy with which changes in the joint angle should be detected by the spanning spindles. However, the sensitivity of a spindle to changes in joint angle also depends on musculoskeletal architecture (Eqs. 2-5). Spindle sensitivity is improved with an increase in the moment arm of the muscle and a decrease in its fascicle length. Moment arm and fascicle length were used to scale the spindle counts for each muscle spanning each joint in the upper and lower limbs. These values were obtained from a variety of sources (fascicle lengths: An et al., 1981; Brand, 1985; Seireg and Arvikar, 1989; Wood et al., 1989; Bassett et al., 1990; Friederich and Brand, 1990; McGill, 1992; moment arms: An et al., 1979; Brand, 1985; Van Eijden et al., 1985; Seireg and Arvikar, 1989; White et al., 1989; McGill, 1992). To assess the resolution in one plane of motion, the square root of the corrected spindle counts was divided by the DOF available at each joint (see Table 1). Values were plotted based on the distance to the end effector. These distances were determined from anthropometric tables (Drillis and Contini, 1966) and assuming a male height of $175 \mathrm{~cm}$.

Using the generic, three-segment model to estimate error transmission from spindles to different coordinate frames provides insight into general properties of sensory transformations, but it does not reflect the special features and consequences of actual musculoskeletal morphometry. Therefore, we modified the generic limb model according to anthropometric parameters. The human elbow and shoulder joints were taken as the model system because these joints have been used extensively for psychophysical studies. These joints were assumed to act only in flexion and extension; that is, the model has only 1 DOF at each joint. The spindle scaling parameters, $k_{1}, k_{2}, k_{3}$, and $k_{4}$, for the model were taken as the average moment arm for each muscle group divided by their average fascicle length. The finger flexor muscles that also span the elbow were excluded because of their dominant role in finger movement. The length of the distal and proximal segments was set to $33 \mathrm{~cm}$ based on anthropometric data (Drillis and Contini, 1966).

\section{Results}

\section{Idealized two-joint system}

The error transmitted from the muscle spindles is dependent on how the spindles are distributed among the muscles. Minimal error is not based on an even distribution of spindles in all three muscles. The shape of the error function and particularly the location of its minimum depend greatly on the coordinates of the limb reference frame in which the spindle errors are transformed. In all results, only a single minimum was found for the distribution of spindles that minimized the error transmitted for each of the reference frames. The transmitted error grew exponentially for spindle distributions that deviated from the optimal.

For a joint angle reference frame $(\phi)$, the error transmitted to an individual joint angle was minimized when all of the spindles were located in the spanning monoarticular muscle (Fig. $3 A, B$ ). However, a joint coordinate system combines both joint angles 
END EFFECTOR ERRORS FOR DIFFERENT SPINDLE DISTRIBUTIONS
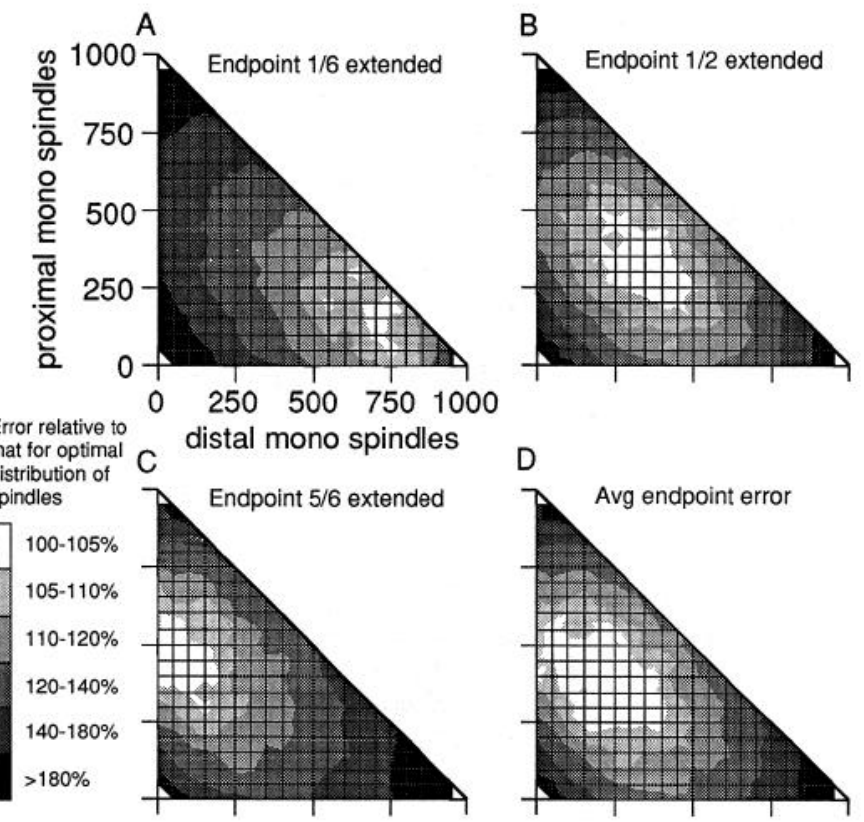

Figure 4. Similar distributions of spindles as shown in Figure 3, but plotting relative error in the estimation of the position of the end of the linkage for three different positions of the end-point (in terms of fraction of distance from proximal joint to maximal extension) plus mean performance for six positions distributed over the entire range $(D)$.

to estimate the absolute position of the distal segment. The least squares error transmitted to both of these joints predicts minimal error is transmitted when the monoarticular muscles contain roughly equal numbers of spindles, while few spindles are located in the biarticular (Fig. $3 C$ ). For a reference frame based on segment angle $(\theta)$, the optimal solution is dramatically different. Minimal error for the distal segment angle locates almost all of the spindles in the biarticular muscle (Fig. $3 E$ ). The combined error in the two segment angles suggests that the proximal monoarticular and the biarticular muscles should contain most of the spindles, and only a few spindles are necessary in the distal monoarticular (Fig. $3 F$ ).

Perhaps the most interesting coordinate frame is end-point cartesian coordinates (Fig. 4). The error transmitted from spindle noise into these coordinates is highly dependent on the initial orientation of the limb. When the arm is nearly fully extended, the optimal solution biases toward spindles located within the proximal monoarticular and the biarticular muscles (Fig. $4 C$ ). This can be explained in part by the fact that an error in the estimated orientation of the proximal joint has a greater affect on end-point error than does an error in the distal joint. As the limb end-point approaches the body, the minimal error transmitted to the end-point occurs when most spindles are in the distal monoarticular muscle (Fig. 4A). The decreased distance from the shoulder joint to the end-point when the distal joint is flexed reduces the end-point error from spindles spanning the proximal joint. Minimizing the mean error transmitted from the spindles to the end-point coordinates for all of the six initial arm positions tested requires all three muscles to contain roughly equal numbers of spindles (Fig. $4 D$ ).

Quite surprising results were found for the second limb model
POSITION AND ORIENTATION ERRORS FOR MODEL 2
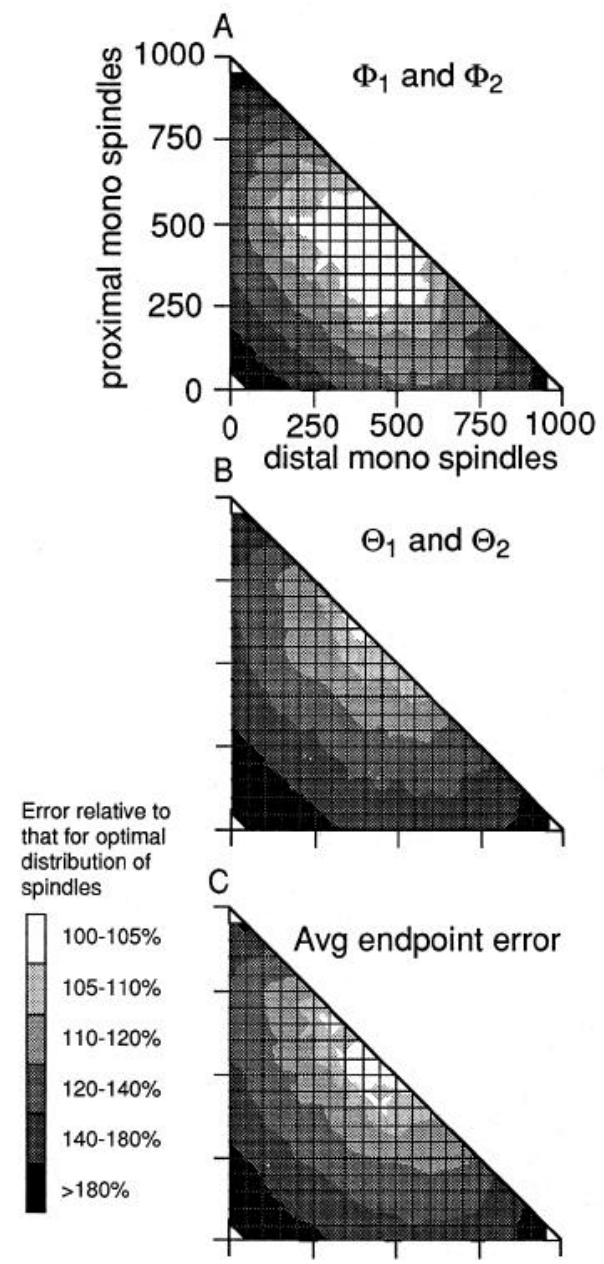

Figure 5. Similar distributions of spindles as shown in Figures 3 and 4 , but for muscles arranged as in model 2 of Figure 2. Note that the optimal distributions of spindles for all three coordinate frames call for no sensors in the biarticular muscle for this topology.

in which the biarticular spanned opposite sides of each joint. For all three coordinate frames, the optimal solution located all of the spindles only in the two monoarticular muscles (Fig. 5). Distributing any of the spindles to the biarticular muscle enhanced rather than dampened the error transmitted to the different reference frames, as illustrated in Figure 6. For the major joints of human limbs, sartorius is the only biarticular muscle that crosses on opposite sides of each spanned joint.

\section{Human spindle distribution}

There is a weak relationship between muscle mass and spindle counts $(r=0.32, p<0.01)$ when the entire population of muscles located with the human body is evaluated (Fig. 7). Larger muscles tend to have more spindles. However, the $\log / \log$ scale in Figure 6 compresses dramatically the variability between parameters; in fact, a muscle's mass is a fairly poor indicator of its spindle count. A stronger relationship exists between spindle counts about a joint and the distance to the end of each limb $(r=0.85, p<0.01 ;$ Fig. 8). The greater the distance from the end effector, the greater the number of spindles acting about the joint (or joint set). These relationships are not altered significantly when the spindle counts are corrected for muscle moment 

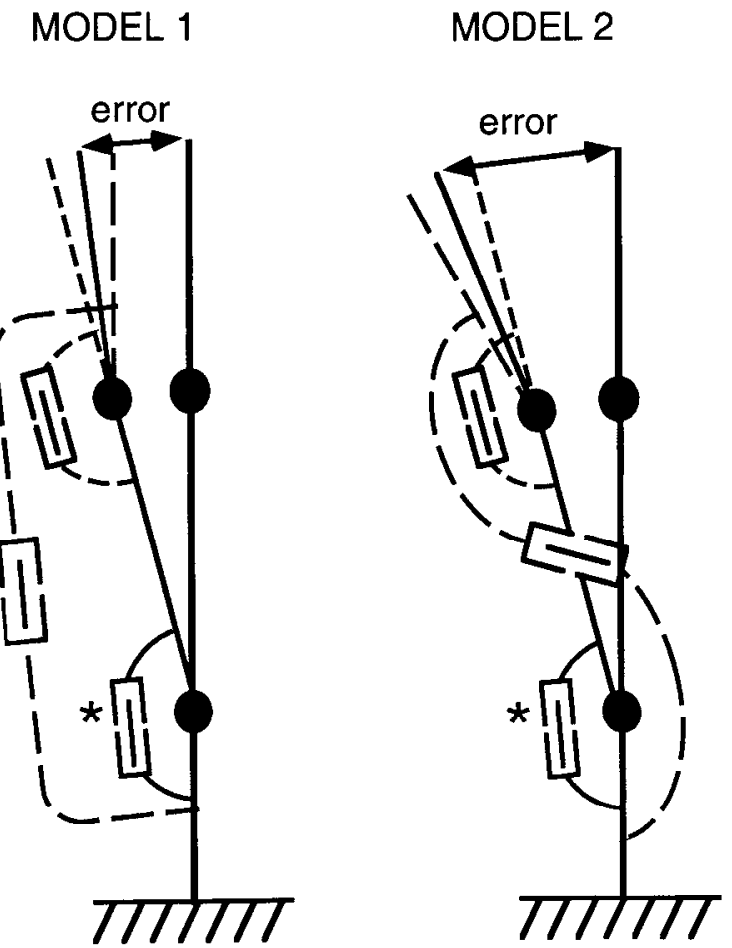

Figure 6. Graphical explanation of the results of Figure 5, suggesting that length sensors in biarticular muscles that cross to opposite sides of a planar linkage (MODEL 2) provide little useful information for determining limb position. For end-point error shown here, consider the use of mono- and biarticular muscle information to compensate for an erroneous signal from the proximal monoarticular sensor (* and associated limb position deviated to the left of the correct, fully extended position). In MODEL 1, correct information from the distal monoarticular muscle (short-dashed line) is averaged with the inference about the distal joint angle derived from the correct length of the biarticular muscle (long-dashed line) and the erroneous information about the proximal joint angle $(*$, solid line), resulting in a reduction of the end-point error. In MODEL 2 , the same averaging process results in an even larger end-point error because the original error $\left({ }^{*}\right)$ leads to the inference that the distal joint must be further flexed away from the midline in order to account for the fact that the biarticular sensor has not responded to the apparent flexion of the proximal joint.

arms, fascicle lengths, or degrees of freedom available at each joint. These results suggest that the resolution of joint angle estimates should be higher in proximal than in distal joints.

The cervical column has many more spindles at each joint in comparison to joints in the upper and lower limbs. This might be expected because integration of any proprioceptive information from the limbs with visual and vestibular signals must be transformed into a global head or body coordinate frame. The gradient between upper and lower cervical resolution is consistent with the body-centered (as opposed to head-centered) coordinate frame that is implicit in Figure 8, but the uncertainties involved in grouping the cervical muscles and joints into these two discrete entities make it unwise to draw any firm conclusions from this. The sense of position in the joints of the lower limb would be expected to be more precise than in the joints of the upper limb.

\section{Human performance}

The precision of position sense predicted by the distribution of muscle spindles within the body was compared to psychophysical studies in the literature (Fig. 9). Based on the distribution

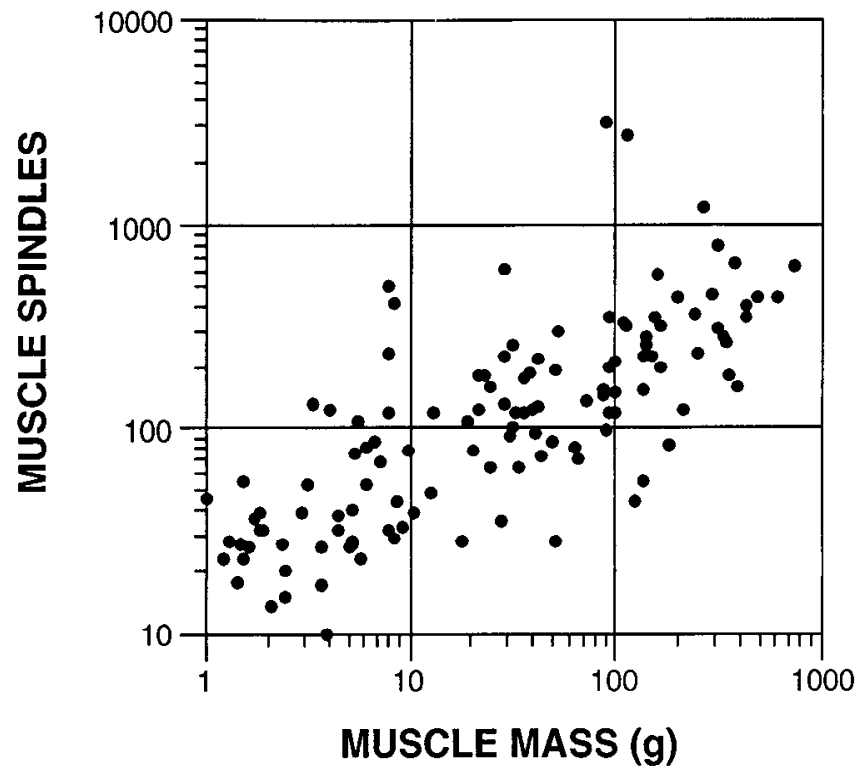

Figure 7. Distribution of spindles in various human muscles, shown as $\log$ of spindle count versus $\log$ of muscle mass in grams.
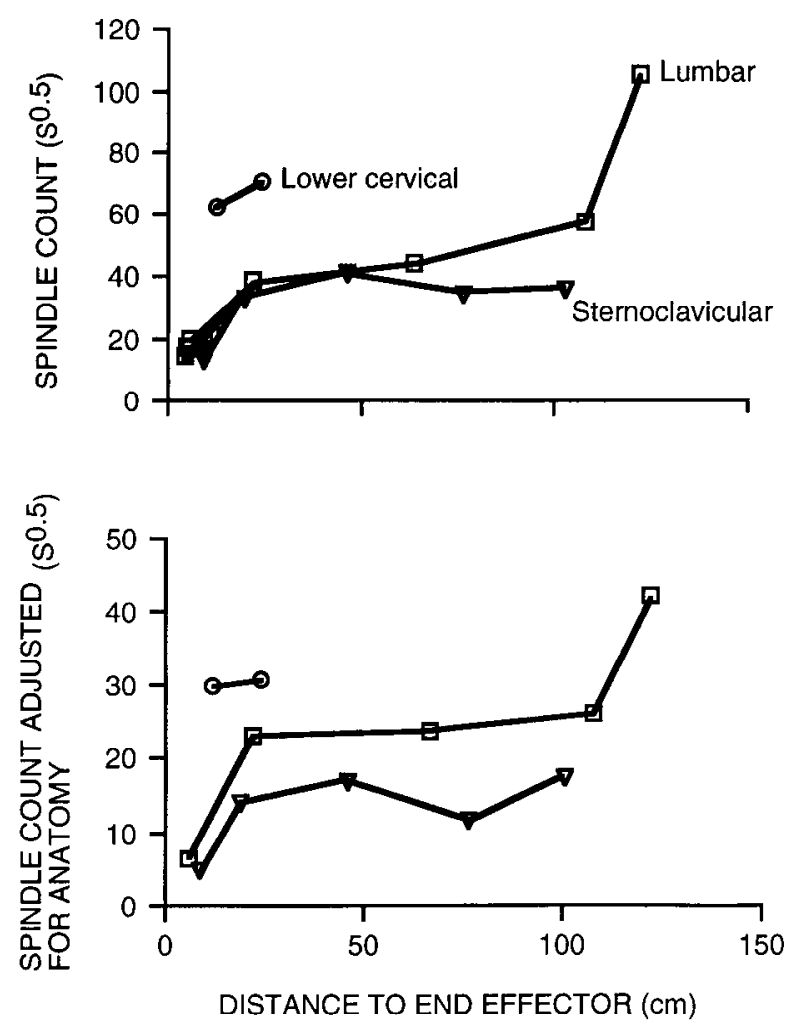

Figure 8. Distribution of spindles crossing various joints in the human versus distance of those joints from the end of the appendage, assuming a body-centered frame with head, arm, and leg as appendages. The squares denote the distribution of spindles about the lumbar, hip, knee, ankle, and toe joints or joint systems (from right to left, proximal to distal, respectively). In a similar manner, the triangles denote the distribution of spindles about the sternoclavicular, shoulder, elbow, wrist, and finger joints or joint systems. The circles denote the lower and upper cervical joint systems. Top, Square root of raw spindle counts (note that points representing the various fingers and toes are all virtually superimposed at left). Bottom, Square root of spindle counts corrected for moment arm and fascicle length of each muscle (only index finger and great toe are included distally). 

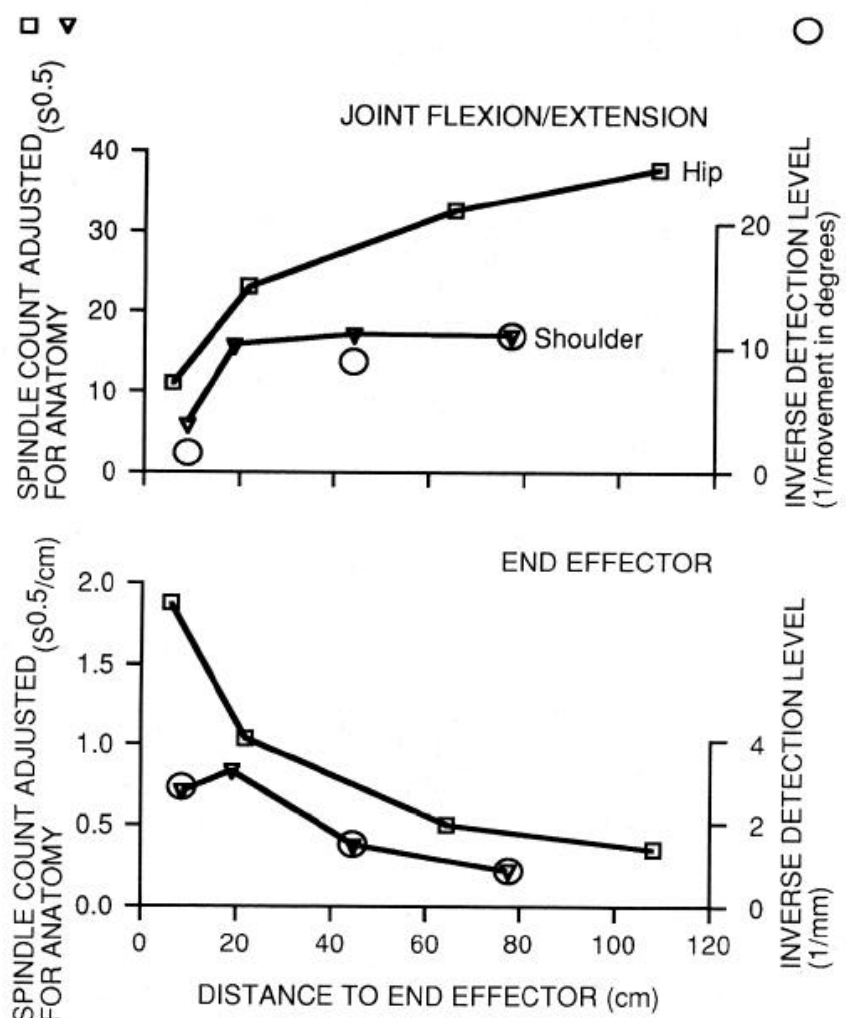

Figure 9. Distribution of spindles (left ordinates) crossing various joints of the arm $(\nabla)$ and leg $(\square)$ in terms of angular deviation of the joint (top) and translational motion at the end-point (bottom). Circles show the corresponding predictions and measured values (from Hall and McCloskey, 1983) of relative position sensitivity (right ordinates).

of muscle spindles, the shoulder, elbow, and wrist would have approximately equal resolution in terms of joint angles $(\phi)$, whereas the accuracy would be much lower at the finger (metacarpophalangeal) joint (Fig. 9, top). In the lower limb, the accuracy in detecting joint angle should diminish gradually from proximal to more distal joints. However, the increased accuracy at the proximal joints would still undercompensate for their longer distance to the end of their limbs in terms of detecting linear motion of the end effector (Fig. 9, bottom). In both limbs, translational movement at the end of the limb would be best detected by the more distal joints despite their low numbers of spindles and consequently low angular resolution.

The validity of these inferences for the arm is supported in a psychophysical study by Hall and McCloskey (1983), which determined the detectability of movement at different joints of the upper limb. They found that detection of angular movement at the elbow and shoulder were similar, whereas the metacarpophalangeal joint was much poorer at the detection of joint motion. However, when the joint movement was converted to movement of the end effector, the opposite result occurred. Note that both the predictions based on the distribution of muscle spindles and the psychophysical experiments assume a fully extended limb. In a more natural working posture, the straightline distance from a joint to the end effector is less than the sum of the limb segment lengths. For the upper limb, the elbow and shoulder may be equidistant to the hand. In this posture, the actual spindle distributions are close to permitting proprioception at each of these joints to contribute equally to end-effector

\section{POSITION AN" ORIENTATION ERRORS FOR HUMAN UPPER LIMB}
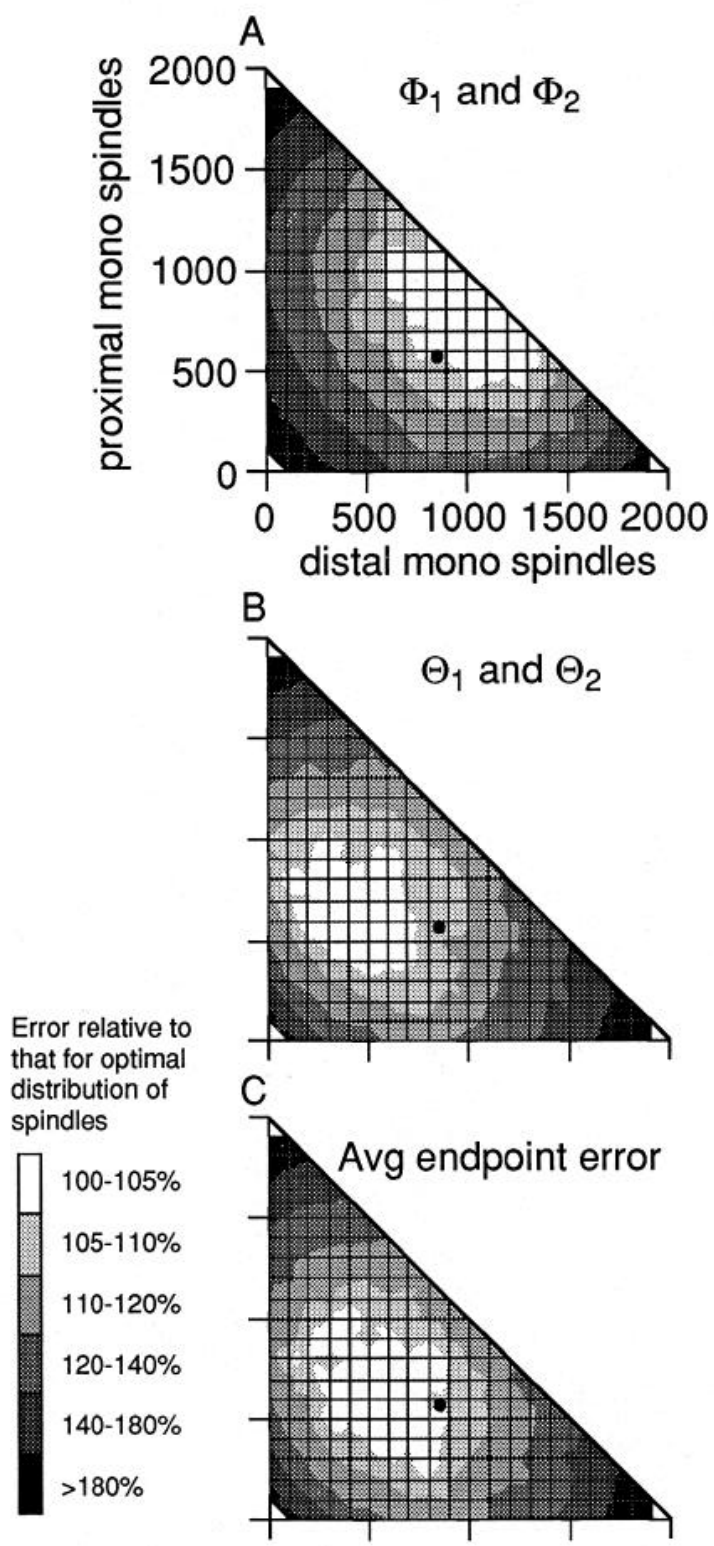

Figure 10. Two thousand spindles reflecting the normal complemen: crossing the human shoulder and elbow joint were distributed variously in an anthropomorphically scaled version of model 1 (Fig. 2) and the relative position errors were determined for the three coordinate frames shown. The black dot in the center of each figure represents the actual distribution of spindles about the human shoulder and elbow joints, which lies near but not at the different minimal error distributions predicted for the model for each coordinate frame.

position sense (see Discussion). Fragmentary data for the ankle (Clark et al., 1985) and knee (Clark et al., 1979), however, suggest a gradient opposite to that predicted in Figure 9. Further experimental measurements on the accuracy of position sense at different joints under similar conditions would be beneficial.

The transmission of spindle error was dependent on the selected reference frame for the limb model with anthropometric elbow and shoulder (Fig. 10). The results are similar to the previous generic model, but there are subtle differences. The segment angle $(\theta)$ and end-point reference frames both favor a 
more even distribution of the spindles among the three muscles as compared to the generic model. However, there is almost no change in the optimal solution in the joint angle reference frame $(\phi)$. The actual distribution of spindles acting about the human elbow and shoulder should result in only $5 \%$ more error in each reference frame than would be produced by an optimal distribution for that frame (shown as black dots in Fig. 10).

The errors transmitted by the anthropometric arm model were tested using the anthropometric spindle distribution. Figure 11 shows the scatterplot of estimated end-point positions for different postures. At full extension, the errors are restricted to a small arc of a circle, the only positions that are possible in this posture. As the arm is retracted, the error distribution becomes circular and smaller in maximal extent. At very close range, the region of errors begins to elongate and rotate about the elbow joint axis. The standard deviation of the end-point errors predicted for the hand are around $0.5 \mathrm{~cm}$. In the angular coordinate frames, the error for the distal joint angle $(\phi, \mathrm{SD}=$ $\left.0.68^{\circ}\right)$ is slightly smaller than for the distal segment angle $(\theta, \mathrm{SD}$ $=0.80^{\circ}$ ). If the distal segment angle is computed indirectly from the two joint angles, then it will be noisier than if computed directly from the spindle signals ( $\mathrm{SD}=0.87$ vs 0.80 ). The $a b-$ solute magnitude of the errors in any of these reference frames is generally lower than actual errors that have been measured in psychophysical studies. The standard deviation of estimating either joint angle or segment angle is generally about 6-10 (Soechting and Ross, 1984; Inglis et al., 1991). Our results are approximately only $10 \%$ of these actual errors, suggesting that (1) spindles may be noisier than we have assumed; (2) fusimotor set may not be fully optimized by subjects under the conditions of a brief psychophysical test session; and/or (3) substantial amounts of noise may be added by the neural computation on the afferent signals.

\section{Discussion}

We have developed a set of mathematical models describing the transductive and computational processes involved in the sense of body position. To derive useful insights into the biological problem and its solution by the nervous system, it is not necessary that the nervous system embody the particular transformations that we have selected for our models. Any general solution based on the premises outlined below should have the same emergent properties as are described in the conclusions outlined below.

Premise 1: muscle spindles provide the majority of the afferent information for position sense under normal circumstances

The psychophysical data supporting this notion have been reviewed elsewhere (Gandevia et al., 1992). The plots in Figure 9 indicate that a model of position sense based solely on muscle spindles makes predictions that are consistent with those data. Although the psychophysical data are based on motion sense, the relative accuracy for sensing orientation at these joints is probably similar. In fact, motion sense data are probably more reliable than position data because of the problem of drift, but the extension of our spindle model to account for velocity signals entails additional assumptions about reference frames and fusimotor control and deconvolution. Meanwhile, more systematic data on position sense in the lower limb and axial joints would be useful.

\section{ENDPOINT ERRORS (SD in $\mathrm{cm}$ )}

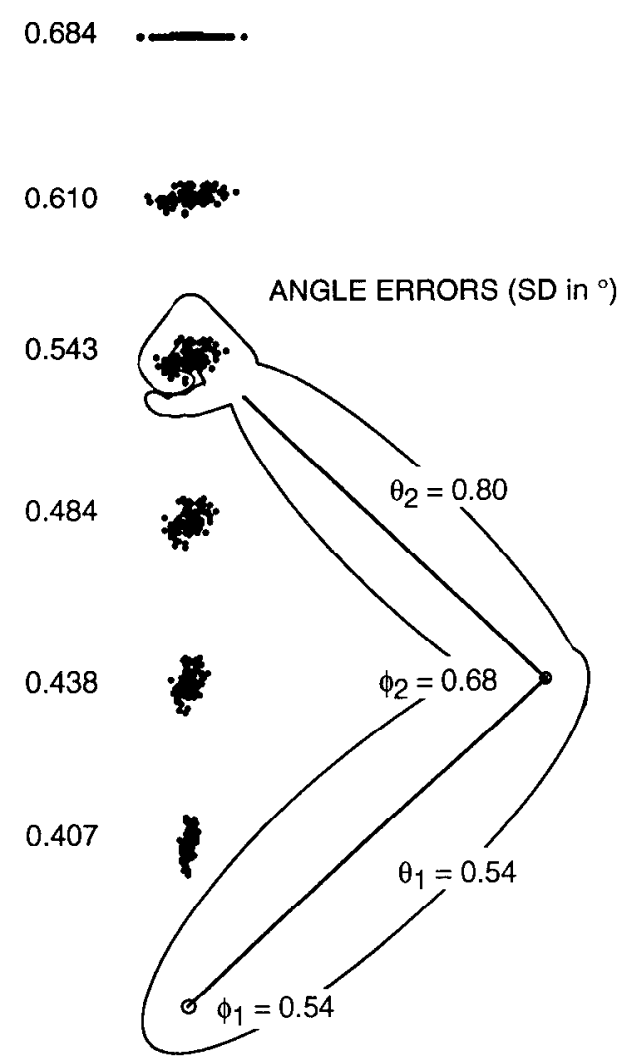

Figure 11. The actual errors in estimated arm position were predicted for a human arm with the known distribution of noisy sensors; numeric values are all standard deviations. Note that the magnitude and particularly the distribution of end-point errors were dependent on the actual posture (six different positions shown here); the angular errors for both segmental orientation $(\theta)$ and intersegmental angle $(\phi)$ were not sensitive to posture.

Premise 2: given any particular set of afferent signals and a desired coordinate frame, the nervous system makes optimal use of the information available to it

This premise is deeply embedded in most theoretical work regarding perception, particularly when considering plasticity or self-organization in neural networks. It has not been generally accepted for proprioception, however, which has historical roots in motor control rather than perception. Originally, muscle spindles were seen as providing length and velocity feedback exclusively or primarily for the control of the muscle in which they resided. Certainly this is one important role played by these sense organs, as evidenced by the tendon jerk reflex, but stopping there is akin to describing the retinal photoreceptors as the sense organ responsible for visual tracking. More recently, there has been growing recognition of the contribution of spindle afferent information both to widespread regulation of heteronymous muscles (McCrea, 1986) and to the composite senses of posture and kinesthesia. Both of these are general problems for which one might expect the nervous system to apply general information processing strategies that would allow for some degree of adaptive optimization. Recently, optimal control theory has been applied to understanding the more general aspects of spinal cord circuitry related to proprioceptive feedback (He et al., 1991; 
Loeb et al., 1990). This article presents an analogous approach to the study of purely perceptual function associated with the ascending projections of many of the same proprioceptors. In fact, one important reason for developing this model is that the application of optimal control theory requires a formal description of the process whereby state variables that are not directly observed can be estimated from the signals of those sensors that are available.

This premise would also account for the effects of afferent microstimulation on the sense of joint position (Macefield et al., 1990) and perhaps for some kinesthetic illusions related to efference (Feldman and Latash, 1982). It seems paradoxical that microstimulation of cutaneous and joint afferents produces illusory motion while microstimulation of spindle afferents does not. This may be explained by noting the relatively large numbers of individual spindles whose signals are averaged to estimate position at a given joint, thereby reducing noise produced by fusimotor and extrafusal mechanical activity and vitiating the effects of microstimulation of a single afferent. Only one Golgi tendon organ was microstimulated, but that did produce illusory motion in a direction consistent with muscle lengthening; this may reflect the fact the tendon organ signals must be summed rather than averaged to estimate active muscle tension (Crago, 1982). Active force in a muscle will stretch elastic components in series with both the muscle fascicles and the spindles, an effect for which the nervous system must correct in order to preserve kinesthetic accuracy. Interestingly, the number of tendon organs appears to covary with the number of spindles in a given muscle (Gandevia et al., 1992). If the tendon organs are used to correct proprioception for tendon compliance, their numbers should depend on both the resolution of the spindle signals (i.e., number of spindle afferents) and the length of the series elastic tissue.

Premise 3: the nervous system can and does use the fusimotor system to achieve a similar goal in all spindles-adjusting sensor gain to match the kinematics expected during a particular sensorimotor behavior

The underlying data and rationale for this premise are discussed in the rationale for our spindle model (see Materials and Methods, and Premise 2). If this or a similarly global summary of fusimotor strategy is not true, then the information processing related to position sense cannot be understood in the absence of a complete and systematic description of spindle activity in each individual muscle. Furthermore, these descriptions would have to be specific to each particular task in which position sense is being used because at least one corollary of this premise is known to be true: the length and velocity sensitivity of the spindles in a given muscle are, in fact, modulated by the fusimotor system according to the task at hand (Loeb and Hoffer, 1985; Loeb et al., 1985; Prochazka et al., 1988).

The original "motor" focus of research on proprioception notcd abovc coincided with great interest in the significance and mechanisms underlying the orderly recruitment of the $\alpha$ motoneurons innervating the extrafusal muscle fibers (Henneman et al., 1974). This conjunction gave rise to various "coactivation" theories of control for the various types of $\gamma$ motoneurons innervating the intrafusal muscle fibers (Vallbo, 1974). Recordings from spindle afferents and fusimotor neurons during a variety of conditions have since suggested that no single pattern of $\alpha-\gamma$ control could account for the modulation of spindle transduction under all of the different conditions of length, velocity, and extrafusal activation that were observed (Loeb, 1984; Prochazka, 1985; Prochazka et al., 1989; Murphy and Martin, 1993). It has been suggested elsewhere that all of these observations can be explained by the general strategy stated in the premise above and that this strategy can be derived independently from signal theory (Loeb, 1984; Loeb and Marks, 1985). As noted in the description of the spindle model (Materials and Methods), any reasonable variation on this strategy is unlikely to have much effect on conclusions regarding static proprioceptors (e.g., spindle secondary endings) and position sense, but the extension of this strategy to dynamic proprioceptors (i.e., spindle primary afferents) and velocity sense will require additional assumptions whose implications have yet to be fully considered. In particular, it would need to account for the inherent asymmetry of shortening versus lengthening muscles and sensors (Inglis et al., 1991).

The task dependence of fusimotor control has important implications for the design and interpretation of psychophysical experiments on the resolution of position and velocity sense. If the complete premise stated above is true, then one would expect the resolution that the subject obtains to depend on the subject's understanding of the range over which he or she will be tested. If the experiment involves the random repositioning of the joint over its entire range of motion, then the subject should select a low fusimotor gain that will avoid saturation of the spindle activity near the extremes of motion; this will, incidentally, magnify the effects of noise in the spindle signal. If the experiment requires judgements over only a small part of the range, then the fusimotor gain can be adjusted so that the entire dynamic range of afferent activity is brought to bear on that part of the range. This would account for the apparent hyperacuity that subjects achieve when making judgements about thickness of grasped objects (John et al., 1989). It may also account for the general observation that active use of a muscle is usually accompanied by some form of fusimotor coactivation (Murphy and Martin, 1993) because the subject would have more specific expectations during self-directed movements.

Conclusion 1: the distribution of spindles among muscles seems better related to the need for information about the position of joints spanned by those muscles than to the control of the muscles themselves

As reported by Banks and Stacey (1988) and drawn in Figure 7 , there is a weak correlation between muscle mass and spindle counts $(r=0.32)$. Nevertheless, spindle density varies enormously and with no obvious systematic trend from neck muscles with 100 spindles/gram to digit muscles with 10 spindles/gram to limb muscles with 1 spindle/gram, and wide ranges within each of those categories. Several reasons may be put forward for expecting some correlation:(1) large muscles produce more force, so noise in their homonymous feedback control would be more problematic; (2) large muscles are more likely to have neuromuscular compartments requiring partitioned control for stability (Windhorst et al., 1989); (3) large muscles have more room for spindles.

The conclusion of the present study is that the overall distribution of spindles accounts well for the proximodistal gradient of accuracy in joint position sense that has been noted in psychophysical studies of the arm (Hall et al., 1983; Clark, 1992). Many details remain unexplained regarding the distribution of spindles among individual muscles and within neuromuscular compartments and histochemically distinct regions of those muscles (e.g., Meyer-Lohmann et al., 1974). It seems 
possible that some of these details can also be explained by a similar appeal to information theory that is outside the scope of this article. For example, selective distribution of spindles and Golgi tendon organs in the slow-fiber regions of some muscles (Richmond and Stuart, 1985) may shield them from sudden mechanical impulses produced by intermittent recruitment of large, fast muscle units.

\section{Conclusion 2: the distribution of spindles among joints does not unambiguously favor any single coordinate frame for position sense}

Onc reason for developing this model-based analysis was the hope that it would provide evidence for accepting or rejecting some of the putative coordinate frames that have been proposed. While there are other coordinate frames beyond those analyzed here, no such unambiguous answer seems likely to emerge. It is possible that this reflects the cumulative effects of uncertainties in many parameters and functional assumptions that went into the model, but our experience with a number of variants of the model suggests that its output is relatively robust in the face of changes in the spindle data and that the trends generally reflect fundamental geometric relationships between the mechanical configuration of the musculoskeletal system and the chosen coordinate frame.

The results presented here suggest that the distribution of spindle afferents may reflect a compromise that is suitable for computing position in several different coordinate frames (Stein, 1992) and particularly in the postures and tasks that are most important to the subject. In this regard, the relatively recent evolution of upright, bipedal posture and fine toolmaking by humans provides special opportunities to examine the behavior of a system that may retain a distribution of sensors that is suboptimal for its currently dominant tasks. Humans normally balance on fully extended legs by detecting body sway with the feet fixed, a problem that would be well addressed by high angular sensitivity in the ankles and perhaps toes as well as hips, and by special sensitivity to locking of the knees at one end of their range of motion. Conversely, manipulation of small objects is usually performed in a preferred arm posture in which equiangular resolution at the elbow and shoulder would contribute equally to end-point resolution. Systematic surveys of spindle distributions and psychophysical data are, unfortunately, not available in animals other than humans. It seems likely, however, that the resolution of position and motion sense may be a limiting factor in at least some motor tasks and that the CNS selects motor strategies according to this constraint instead of or in addition to constraints such as minimizing mechanical work or stress.

It is worth noting that all of the putative coordinate frames that we and others have considered are orthogonal, perhaps because we lack mathematical tools for dealing with nonorthogonal (highly redundant) coordinate frames. It is possible, however, that the intermediate coordinate frame is nonorthogonal. In the extreme, it could be based entirely on the natural coordinates of the sensors themselves, with little or no subsequent computation. This would obviate the need for explicit computations, for example, to separate frames based on position and velocity (Prud'Homme and Kalaska, 1994). The distribution of sensors actually present would then reflect the importance of their signals for the complete behavioral repertoire of the whole animal, a situation that would tax the sort of inverse analysis presented here.

\section{References}

Amonoo-Kuofi HS (1983) The density of muscle spindles in the medial, intermediate and lateral columns of human intrinsic postvertebral muscles. J Anat 136:509-519.

An KN, Chao EY, Cooney WP, Linsheid RL (1979) Normative model of human hand for biomechanical analysis. J Biomech 12:775-788.

An KN, Hui FC, Morrey BF, Linscheid RL, Chao EY (1981) Muscles across the elbow joint: a biomechanical analysis. J Biomech 14:659669.

Banks R, Stacey M (1988) Quantitative studies on mammalian muscle spindles and their sensory innervation. In: Mechanoreceptors, pp 263269. Plenum.

Bassett RW, Browne AO, Morrey BF, An KN (1990) Glenohumoral muscle force and moment mechanics in a position of shoulder instability. J Biomech 23:405-415.

Brand PW (1985) Clinical mechanics of the hand. St. Louis, MO: Mosby.

Burke D, Hagbarth KE, Skuse NF (1979) Voluntary activation of spindle endings in human muscles temporarily paralysed by nerve pressure. J Physiol (Lond) 287:329-336.

Caminiti R, Johnson PB, Galli C, Ferrania S, Burnod Y (1991) Making arm movements within different parts of space: the premotor and motor cortical representation of a coordinate system for reaching to visual targets. J Neurosci 11:1182-1197.

Clark FJ (1992) How accurately can we perceive the positions of our limbs? Behav Brain Sci 15:725-726.

Clark FJ, Horch KW, Bach SM, Larson GF (1979) Contributions of cutaneous and joint receptors to static knee-position sense in human. J Neurophysiol 42:877-888.

Clark FJ, Burgess RC, Chapin JW, Lipscomb WT (1985) Role of intramuscular receptors in the awareness of limb position. J Neurophysiol 54:1529-1540.

Clark FJ, Burgess RC, Chapin JW (1986) Proprioception with the proximal interphalangeal joint of the index finger. Brain 109:11951208.

Crago PE, Houk JC, Rymer WZ (1982) Sampling of total muscle force by tendon organs. J Neurophysiol 47:1069-1083.

Drillis R, Contini R (1966) Body segment parameters. Washington, DC: Office of Vocational Rehabilitation, Department of Health and Education Welfare.

Feldman AG, Latash ML (1982) Afferent and efferent components of joint position sense; interpretation of kinaesthetic illusion. Biol Cybern 42:205-214.

Friederich JA, Brand RA (1990) Technical note: muscle fiber architecture in the human lower limb. J Biomech 23:91-95.

Gandevia S, McCloskey DI, Burke D (1992) Kinaesthetic signals and muscle contraction. Trends Neurosci 15:62-65.

Georgopoulos AP, Schwartz AB, Kettner RE (1986) Neuronal population coding of movement direction. Science 233:1357-1460.

Hall LA, McCloskey DI (1983) Detections of movements imposed on finger, elbow and shoulder joints. J Physiol (Lond) 335:519-533.

Hasan Z (1983) A model of spindle afferent response to muscle stretch. J Neurophysiol 49:989-1006.

He J, Levine WS, Loeb GE (1991) Feedback gains for correcting small perturbations to standing posture. IEEE Trans AC 36:322-332.

Henneman E, Clamann HP, Gillies JD, Skinner RD (1974) Rank order of motoneurons within a pool: law of combination. J Neurophysiol 34:1338-1349.

Hogg RV, Tanis EA (1977) Probability and statistical inference. New York: Macmillan.

Hulliger M (1984) The mammalian muscle spindle and its central control. Rev Physiol Biochem Pharmacol 101:1-110.

Inglis JT, Frank JS, Inglis B (1991) The effect of muscle vibration on human position sense during movements controlled by lengthening muscle contraction. Exp Brain Res 84:631-634.

John KT, Goodwin AW, Darian-Smith I (1989) Tactile discrimination of thickness. Exp Brain Res 78:62-68.

Loeb GE (1984) The control and responses of mammalian muscle spindles during normally executed motor lasks. Exercise Sport Sci Rev 12:157-204.

Loeb GE, Hoffer JA (1985) The activity of spindle afferents from cat anterior thigh muscles. II. Effects of fusimotor blockade. $J$ Neurophysiol 54:565-577.

Loeb GE, Marks WB (1985) Optimal control principles for sensory 
transducers. In: Proceedings of the international symposium: the muscle spindle (Boyd IA, Gladden MH, eds), pp 409-415. London: Macmillan.

Loeb GE, Hoffer JA, Pratt CA (1985) The activity of spindle afferents from cat anterior thigh muscles. I. Identification and patterns during normal locomotion. J Neurophysiol 54:549-564.

Loeb GE, Levine WS, He J (1990) Understanding sensorimotor feedback through optimal control. Cold Spring Harbor Symp Quant Biol 55:791-803.

Macefield G, Gandevia S, Burke D (1990) Perceptual responses to microstimulation of single afferents innervating joints, muscle and skin of the human head. J Physiol (Lond) 429:113-129.

Matthews PBC (1972) Mammalian muscle receptors and their central actions. London: Arnold.

Matthews PBC (1988) Proprioceptors and their contribution to somatosensory mapping: complex messages require complex processing. Can J Physiol Pharmacol 66:430-438.

McCrea DA (1986) Spinal cord circuitry and motor reflexes. Exercise Sport Sci Rev 14:105-142.

McGill SM (1992) A myoelectrically based dynamic three-dimensional model to predict loads on lumbar spine tissues during lateral bending. J Biomech 25:395-414.

Meyer-Lohmann J, Riebold W, Robrecht D (1974) Mechanical influence of the extrafusal muscle on the static behaviour of the de-efferented primary muscle spindle endings in the cat. Pfluegers Arch 352 : $26 \%-278$

Mouchnino L, Aurenty R, Massion J, Pedotti A (1993) Is the trunk a reference frame for calculating leg position? J Neurobiol, in press.

Murphy PR, Martin HA (1993) Fusimotor discharge patterns during rhythmic movements. Trends Neurosci 16:273-278.

Prochazka A (1985) Afferent input during normal movements. In: Feedback and motor control in invertebrates and vertebrates (Barnes WJ, Gladden MH, eds). London: Croom-Helm.

Prochazka A, Hulliger M, Trend P, Durmuller N (1988) Dynamic and static fusimotor set in various behavioural contexts. In: Mechanoreceptors (Hnik P, Soukup T, Vejsade R, Zelena J, eds), pp 417-430. New York: Plenumi.

Prochazka A, Trend P, Hulliger M, Vincent S (1989) Ensemble proprioceptive activity in the cat step cycle: towards a representative look-up chart. Prog Brain Res 80:6 1-74.

Prud'Homme MJL, Kalaska JF (1994) Proprioceptive activity in primate primary somatosensory cortex during active arm reaching movements. J Neurophysiol, in press.
Richmond FJR, Stuart DG (1985) Distribution of sensory receptors in the flexor carpi radialis muscle of the cat. J Morphol 183:1-13.

Richmond F.IR, Bakker GI, Bakker DA, Stacey M.J (1986) The innervation of tandem muscle spindles in the cat neck. J Comp Neurol 245:483-497.

Schaafsma A, Otten E, Van Willigen JD (1991) A muscle spindle model for primary afferent firing based on a simulation of intrafusal mechanical events. J Neurophysiol 65:1297-1312.

Seireg A, Arvikar RJ (1989) Biomechanical analysis of musculoskeletal structure for medicine and sports. New York: Hemisphere.

Soechting JF, Flanders M (1992) Moving in three-dimensional space: frames of reference, vectors, and coordinate systems. Annu Rev Neurosci 15:167-191.

Soechting JF, Ross B (1984) Psychophysical determination of coordinate representation of human arm orientation. Neuroscience 13: 595-604.

Stein JF (1992) The representation of egocentric space in the posterior parietal cortex. Behav Brain Sci 15:691-700.

Stein RB (1967) The information capacity of nerve cells using a frequency code. Biophysiology 7:797-826.

Thompson DW (1917) On form and mechanical efficiency. In: On growth and form, pp 670-718. Cambridge: Cambridge UP.

Vallbo AB (1974) Human muscle spindle discharge during isometric voluntary contractions. Amplitude relations between spindle frequency and torque. Acta Physiol Scand 90:319-336.

Van Eijden IMGJ, de Boer E, Weijs WA (1985) The orientation of the distal part of the quadriceps femoris as a function of the knee flexion-extension angle. J Biomech 18:803-809.

Voss H (1971) Tabelle der absoluten und relativen muskelspindelzahlen der menschlichen skelettmuskulatur. Anat Anz 129:562-572.

Wann JP, Ibrahim SF (1992) Does limb proprioception drift? Exp Brain Res 91:162-166.

White SC, Yack HJ, Winter DA (1989) A three-dimensional musculoskeletal model for gait analysis. Anatomical variability estimates. J Biomech 22:885-893.

Windhorst U, Hamm TM, Stuart DG (1989) On the function of muscle and reflex partitioning. Behav Brain Sci 12:629-681.

Wood JE, Meek MG, Jacobsen SC (1989) Quantitation of human shoulder anatomy for prosthetic arm control. II. Anatomy matrices. J Biomech 22:309-325. 